\title{
El «Mundialito shipibo»: identidad, etnicidad y modernidad en la Amazonía peruana*
}

\author{
Oscar Espinosa**
}

Quiero agradecer especialmente a Sanny Ancón con quien hemos venido conversando sobre el Mundialito shipibo desde hace muchos años, y quien, además, es el autor de las fotos que acompañan este artículo.

** Doctor en Antropología, y profesor principal del Departamento de Ciencias Sociales de la Pontificia Universidad Católica del Perú (PUCP). Correo electrónico: oespinosa@pucp.edu.pe

Fecha de recepción: 20/05/20. Fecha de aceptación: 02/10/20. 


\title{
$\mathrm{El}$ «Mundialito shipibo»: identidad, etnicidad y modernidad en la Amazonía peruana
}

\section{ReSUMen}

Desde hace cerca de treinta años se viene celebrando en la ciudad de Pucallpa, en la Amazonía peruana, el campeonato de fútbol conocido como el «Mundialito shipibo», que convoca a familias shipibas provenientes de todas las comunidades nativas ubicadas a lo largo del Ucayali, pero también de distintas partes del país. A través del tiempo, el Mundialito se ha ido transformando, buscando modernizarse y profesionalizarse, aunque puede verse también como una nueva versión del antiguo ani xeati, la gran fiesta tradicional del pueblo shipibo. De ahí que, hoy en día, el Mundialito shipibo no solo es un campeonato deportivo, sino también un espacio donde los parientes se reencuentran, los líderes se reúnen para tomar decisiones juntos, los y las jóvenes se conocen para formar nuevas parejas, se hacen intercambios comerciales, y por supuesto, se come, se bebe, se baila y se celebra.

Palabras clave: fútbol, pueblos indígenas, Amazonía, shipibo-konibo.

\section{The "Mundialito shipibo": identity, ethnicity and modernity in the Peruvian Amazon}

\begin{abstract}
For nearly thirty years, the Mundialito shipibo, a football (soccer) championship has been held in the city of Pucallpa, in the Peruvian Amazon region, bringing together Shipibo-Konibo families from the different communities located along the Ucayali river, but also from other parts of the country as well. Through time, the Mundialito has undergone important changes, trying to become more modern and professional. However, at the same time, the Mundialito can be seen as a new version of the traditional ani xeati, the big feast of the Shipibo people. Therefore, nowadays, the Mundialito is not only a sports event, but also a moment in which relatives get together, indigenous leaders meet, young people get to know each other and form new couples, commercial exchanges are made, and, of course, as in any other big celebration, people eat and drink together, dance and celebrate together.
\end{abstract}

Keywords: football / soccer, indigenous peoples, Amazonia, shipibo-konibo. 


\section{INTRODUCCIÓN}

Desde el año 1992 se viene celebrando en Pucallpa el campeonato deportivo más conocido como «Mundialito shipibo». A lo largo de todo el año, las distintas comunidades del pueblo shipibo-konibo se preparan para este gran acontecimiento, que tiene lugar en los meses de enero o febrero, en medio de la temporada de lluvias, cuando el transporte se hace más rápido en el río Ucayali y cuando los colegios y escuelas están de vacaciones. Pero, además de ser un importante evento deportivo, el Mundialito es un evento complejo y multidimensional en el que no solo se juegan y se ven los partidos de fútbol tanto de hombres como de mujeres, sino que también ocurren muchas otras cosas: los parientes se reencuentran, se forman nuevas parejas, se discuten asuntos políticos, y, por supuesto, se come, se bebe, se baila y se celebra. Es, en una palabra, el evento social más importante para el pueblo shipibo-konibo que, desde distintas partes del país, llega a celebrarlo ${ }^{1}$.

El pueblo shipibo-konibo ha vivido por varios siglos a lo largo del río Ucayali y sus afluentes y proviene de la fusión de por lo menos tres pueblos de lengua pano que vivían en esta región: shipibo, xetebo y konibo. Con el paso del tiempo, estos pueblos fueron integrándose cada vez más entre sí debido no solo a alianzas matrimoniales, sino también por la reducción demográfica de muchas de sus comunidades. Así, hoy en día se consideran parte de una única y gran "nación shipiba» (Espinosa, 2017), y por ello, en este texto los denominaré simplemente como «shipibo».

Siendo conservadores, se podría decir que la población shipiba actual supera las 45000 personas, a pesar de que el último censo nacional realizado en Perú en 2017 indique solo 34000 personas que se han autoidentificado como tal. Es importante mencionar que este censo ha recibido numerosas críticas por la forma en que se ha implementado, y de manera particular se ha criticado la forma en que se aplicó la pregunta por la identidad o adscripción étnica. Asimismo, los datos censales referidos a la autoidentificación étnica no incluyen a la población menor de doce años, y si se toma en consideración que un porcentaje muy alto de la población indígena amazónica en el Perú es menor de edad, el número real podría ser significativamente mayor, y hasta doblarse. Una parte de la población shipiba vive en las más de 150 comunidades nativas ubicadas a lo largo del río Ucayali y sus afluentes, pero también hay un porcentaje importante y creciente que vive en centros urbanos regionales, e incluso en grandes ciudades. Se calcula que aproximadamente una tercera parte de la población shipiba viviría actualmente en la zona urbana de la

\footnotetext{
Las reflexiones aquí presentadas provienen de información recogida a lo largo de casi veinte años. La primera vez que asistí al Mundialito fue en el año 2001. Desde entonces, ha continuado mi fascinación con este evento, y lo he ido siguiendo año a año, de manera presencial o a distancia.
} 
provincia de Coronel Portillo, cuya ciudad principal es Pucallpa y sus alrededores, principalmente en el distrito de Yarinacocha ${ }^{2}$. Asimismo, existen comunidades shipibas urbanas en distintas ciudades amazónicas, como Iquitos, Puerto Maldonado, Tingo María, etc., pero también en grandes ciudades ubicadas en otras regiones del país, como Lima, Arequipa o Ica.

\section{EL FÚTBOL EN EL MUNDO INDÍGENA AMAZÓNICO}

El fútbol se juega hoy en día prácticamente en todos los pueblos indígenas de la Amazonía; sin embargo, lo importante no es que los indígenas amazónicos jueguen al más popular deporte del mundo, sino más bien — como señala Giulianotti (1999) — cómo se usa el fútbol para expresar formas particulares de identidad social y cultural. En este sentido, la práctica indígena del fútbol no tiene que ver tanto con su «esencia» - para usar un término de Giulianotti-, sino con las prácticas culturales de cada sociedad. De ahí que la forma en que se juega el fútbol en distintas sociedades indígenas constituye una expresión, no solo sobre el fútbol, sino en particular sobre las sociedades mismas.

A pesar de que es casi omnipresente en la Amazonía indígena ${ }^{3}$, el número de investigaciones realizadas a profundidad sobre el fútbol y las distintas dimensiones sociales y culturales vinculadas con su práctica sigue siendo muy escaso. En la última década, sin embargo, han comenzado a incrementarse los estudios que se enfocan en distintos aspectos relacionados con la vida social indígena en torno a este deporte. Estos estudios se pueden clasificar de distintas maneras. Una forma de hacerlo sería distinguir entre aquellos que se centran en la práctica del fútbol en una sociedad indígena en particular frente a aquellos que analizan campeonatos entre distintos pueblos o comunidades indígenas. Así, en el primer grupo se encuentran los trabajos de Brito Vianna (2001a y 2008) sobre los xavante, los de Andrade do Nascimento y Faustino (2009) y de Fassheber (2010) sobre los kaingang (2009), de Acuña (2010) sobre los yanomami, Fermino (2012) sobre los laklānô-xokleng, de Pires Rosse (2013) sobre los maxakali (tikmũ'ũn), de Berloffa Rodrigues sobre los kaiowá y guaraní (2013 y 2014), de Nascimento (2015) sobre los mundurukú, así como los estudios de Massa (2009) sobre los matsigenka, de Walker (2013)

\footnotetext{
2 Según el censo de 2017, la población indígena urbana en la provincia de Coronel Portillo es mayor de 12000 personas, y aquí, nuevamente, hay que considerar que no se está incluyendo a la población menor de doce años en esta cifra.

3 Resulta interesante, al respecto, el cuadro que coloca Brito Vianna (2001a) como apéndice en su tesis de maestría, en el que señala todas las referencias puntuales hechas por diversos autores relativas al fútbol indígena en Brasil en trabajos dedicados a otros temas.
} 
sobre los urarina, y de Dziubinska (2013) sobre los kakataibo, estos tres últimos los únicos realizados en la Amazonía peruana.

Con relación a los estudios sobre campeonatos de fútbol indígena, estos aún son menos. Destacan los de Chiquetto (2014) y de Vieira de Souza (2017) sobre el campeonato de fútbol indígena en el contexto del peladão, que es un campeonato de fútbol popular que tiene lugar en la ciudad amazónica de Manaos, en Brasil. Y en referencia al Mundialito shipibo solamente existen referencias de pasada en un artículo de Giancarlo Rolando (2010) y en la tesis de maestría de Carlos Suárez Álvarez (2011). La única tesis que ahonda algo más en el tema es la de licenciatura en Lingüística de Maricielo Guillén (2018) que se centra en las formas lingüísticas propias de la narración de los partidos de fútbol en lengua shipiba.

La mayoría de estos estudios, sin embargo, se enfoca en las dinámicas sociales que se establecen entre distintas comunidades desde tres perspectivas centrales. Uno de los temas principales desarrollados en estos trabajos (Massa, 2009; Fassheber, 2010; Acuña, 2010), así como en aquellos dedicados, más en general a los juegos y deportes indígenas (Vinha, 2004; Almeida y otros, 2010; Grando, 2015, etcétera), tiene que ver con el cuerpo, su lugar privilegiado en los procesos de socialización y de construcción de las personas indígenas. Este tema se conecta, además, de manera simultánea con dos largas tradiciones académicas, aquella que ve el tema de la construcción de los cuerpos en la sociología y antropología del deporte (referencias), así como en la etnología indígena amazónica (Seeger y otros, 1979; Turner, 1995; Conklin, 1996; McCallum, 2001; Vilaça, 2005, etcétera).

Un segundo tema se refiere a la práctica del fútbol en relación con los vínculos y relaciones intra e intercomunitarias. En general, la mayoría de estudios coincide en la importancia del fútbol como espacio de encuentro social. Sin embargo, aquí se tiene que distinguir entre los partidos locales entre dos o más comunidades con motivo de alguna fiesta comunal — como se puede apreciar con detalle en los trabajos de Massa (2009), Acuña (2010) o Dziubinska (2013) - de los grandes campeonatos futbolísticos. A respecto, Brito Vianna (2001b) señala, como un dato importante a tomar en cuenta, que estos últimos se realizan todos en ciudades.

Finalmente, un tercer tema que aparece en estos estudios se refiere a la reconfiguración y actualización de prácticas ancestrales. Así, por ejemplo, Acuña (2010) describe cómo el fútbol se relaciona con las luchas rituales entre los yanomami, o Massa (2009) analiza cómo las habilidades futbolísticas de los jóvenes matsigenka reemplazan en parte a las habilidades tradicionales para la caza o la pesca. 


\section{EL «MUNDIALITO SHIPIBO»}

El Mundialito se jugó por primera vez en 1992. La idea del campeonato surgió de una iniciativa del profesor Brino Pineda Vela, quien enseñaba Educación Física en el Instituto Pedagógico Público Bilingüe de Yarinacocha (ISPPBY). El profesor Pineda veía el interés de los estudiantes shipibos del Pedagógico y lo bien que muchos de ellos jugaban, y pensó que sería una excelente oportunidad, no solo para que puedan demostrar sus habilidades deportivas, sino sobre todo como un espacio de encuentro con las familias y comunidades luego de estar estudiando todo el año lejos de ellos (Wilmer Ancón, comunicación personal, enero 2016 y noviembre 2019). El profesor Pineda recibió un amplio apoyo del director del instituto en ese momento, el profesor Alejandro Ruiz López, quien oficialmente hizo la convocatoria a las comunidades para que participaran en el evento. De ahí que a ambos se les reconozca hoy en día como los «fundadores» del Mundialito.

En el primer campeonato participaron dieciocho equipos, y con el tiempo, el número de equipos se ha ido incrementando. Hacia inicios del siglo XXI eran unos treinta equipos. Hacia fines de la década pasada eran ya más de cincuenta equipos, y en los últimos años vienen participando más de ochenta equipos. Asimismo, se ha ido incrementando el número de equipos de mujeres. Hasta hace poco, solo de manera eventual se organizaban partidos con equipos femeninos en el contexto del Mundialito. Sin embargo, a partir del año 2013 participan de manera constante. En el año 2016 se convocó oficialmente a la primera copa de fútbol femenino, logrando la participación de veintiocho equipos de mujeres. Desde este año, el título oficial del evento explícitamente señala que se trata de un «Campeonato de Fútbol Masculino y Femenino", tal como se puede apreciar también en el comunicado oficial de la AFUIP convocando al campeonato del año 2020.

El nombre del campeonato también ha sufrido varios cambios, y a través de los años ha sido llamado de distinta manera: «Copa de la Amistad», «Mundialito de los Pueblos Indígenas», «Copa Inter Etnias», «Copa EIB (Educación Intercultural Bilingüe)», "Copa Intercultural», "Campeonato Inter Comunidades», "Campeonato de Fútbol Indígena», etc. Sin embargo, es ampliamente conocido simplemente como el «Mundialito shipibo», incluso cuando de cuando en cuando participan también equipos de otros pueblos indígenas.

En los últimos años, el formato que se sigue es básicamente el mismo. El evento comienza con una inauguración formal, en la que participan, además de los organizadores del evento, diversas autoridades invitadas, incluyendo a los dirigentes de las organizaciones indígenas regionales (ver foto 1). En algunos años, también han participado diversas autoridades vinculadas con el Estado, como el alcalde de la Municipalidad Provincial de Coronel Portillo, el presidente del Instituto Peruano del Deporte, y alguno que otro congresista representante de la región Ucayali. 


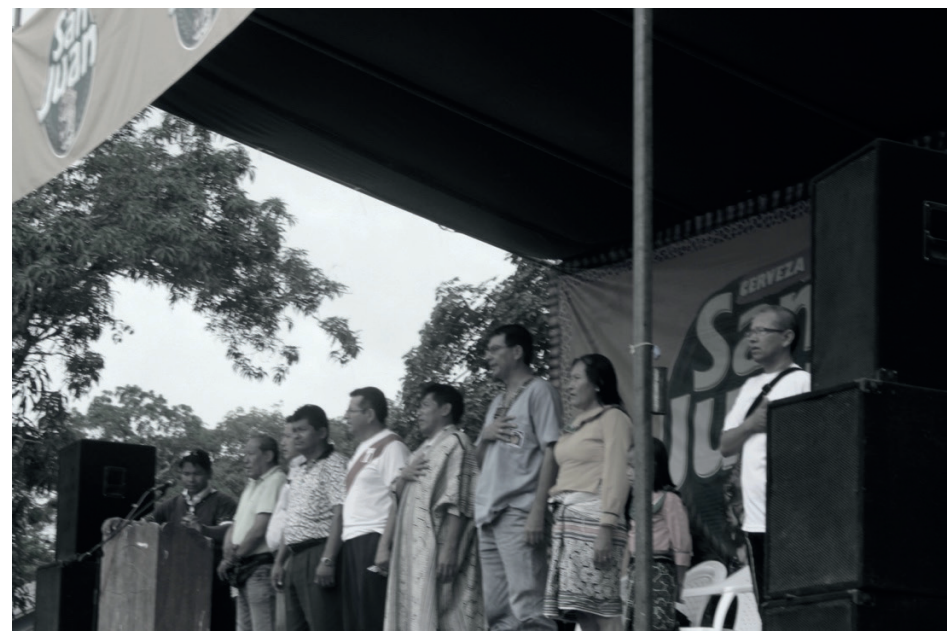

Foto 1. Acto protocolar de inauguración (Foto de Sanny Ancón, 2014)]

Asimismo, diversas organizaciones indígenas se han ido sumando y apoyan la gestión del evento, como la Organización Regional de AIDESEP de Ucayali (ORAU), la Federación de Comunidades Nativas de Ucayali (FECONAU), entre otras. También cuentan con el apoyo oficial del gobierno regional a través de su oficina de asuntos indígenas (IRDECON). En algunas ocasiones, también han recibido otros apoyos institucionales del Estado como del Instituto Peruano del Deporte (IPD), así como de diversas municipalidades locales, como la Municipalidad Provincial de Coronel Portillo, la Municipalidad Distrital de Yarinacocha, la Municipalidad Distrital de Padre Márquez, etc. Finalmente, en los últimos años, también algunos empresarios shipibos han comenzado a colaborar económicamente, como por ejemplo, el maestro Roger Bardales Romaina (Mukan Ranko) o el maestro Roger López (Soi Pino), ambos a cargo de albergues chamánicos prestigiosos.

Tradicionalmente, este campeonato se ha realizado en la sede del Instituto Pedagógico Bilingüe de Yarinacocha, en el llamado «Estadio Oficial de los Pueblos Indígenas Alejandro Ruiz López». Pero en los últimos años también se ha está utilizando el Estadio Municipal del Distrito de Yarinacocha "Jorge Forest Forest» debido a que el estadio del ISPPBY está en remodelación.

El día de la inauguración se comienza con los actos protocolares propios de este tipo de eventos, en los que se canta el himno nacional y las diversas autoridades presentes dan sus palabras de bienvenida. Una vez terminados estos saludos protocolares, se pasa al desfile de inauguración. En este, cada equipo desfila alrededor del campo de juego con su camiseta oficial y acompañado de una delegación de su 
comunidad que incluye, en la medida de lo posible al jefe de la comunidad y también a las reinas de belleza elegidas el año anterior ${ }^{4}$ (ver fotos 2,3 y 4 ).

En un inicio, los equipos usaban camisetas que eran donadas por los auspiciadores como, por ejemplo, las municipalidades. La mayoría de camisetas correspondía a las de selecciones o equipos de fútbol internacionales. Al ser donaciones, los equipos no tenían decisión sobre los colores que les asignaban y, por lo tanto, podían cambiar de año a año. En más de una ocasión, este hecho sirvió también para hacer bromas, como la ocurrencia de un jugador que se pintó el cuerpo con huito (Genipa americana) cuando le tocó jugar con la camiseta de un equipo africano (Isaac Alva, comunicación personal, agosto de 2019). Sin embargo, en los últimos años se trata de mantener la misma camiseta, como ocurre con los equipos profesionales.

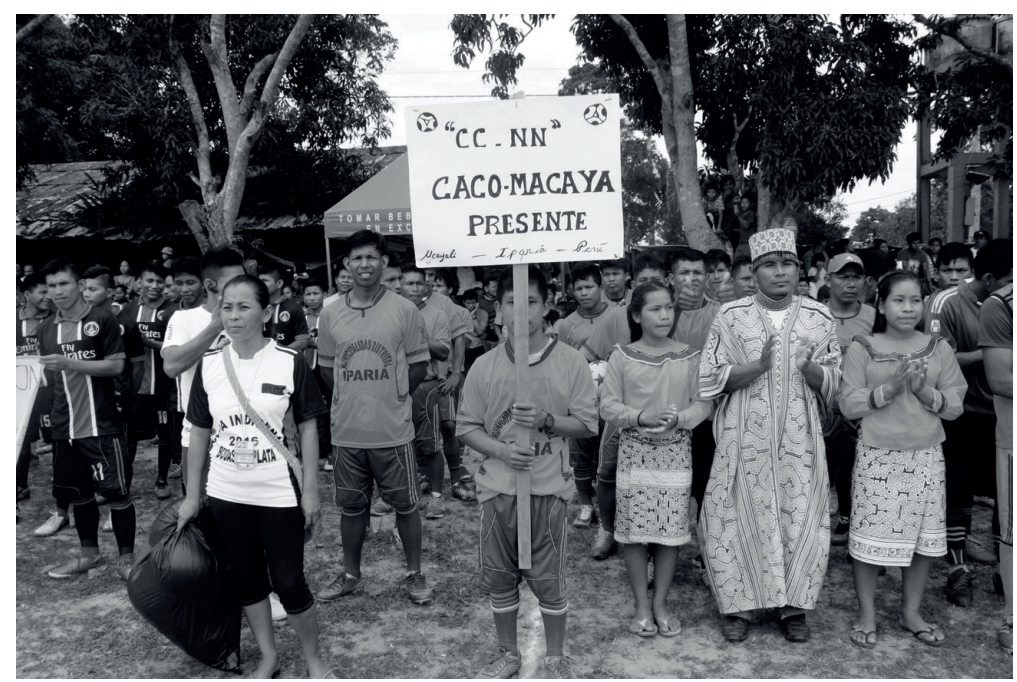

Foto 2. Equipo de comunidad Caco Macaya (Foto de Sanny Ancón 2014).

4 El vínculo entre concursos de belleza y campeonatos deportivos data desde los orígenes de ambos en los Estados Unidos (Banet-Weiser 1999). En un escenario distinto, Schweitzer de Palacios (2008) también encuentra una conexión interesante entre las reinas de belleza y el Mundial de Fútbol, al que se incorpora también a un chamán shuar de la Amazonía ecuatoriana. 


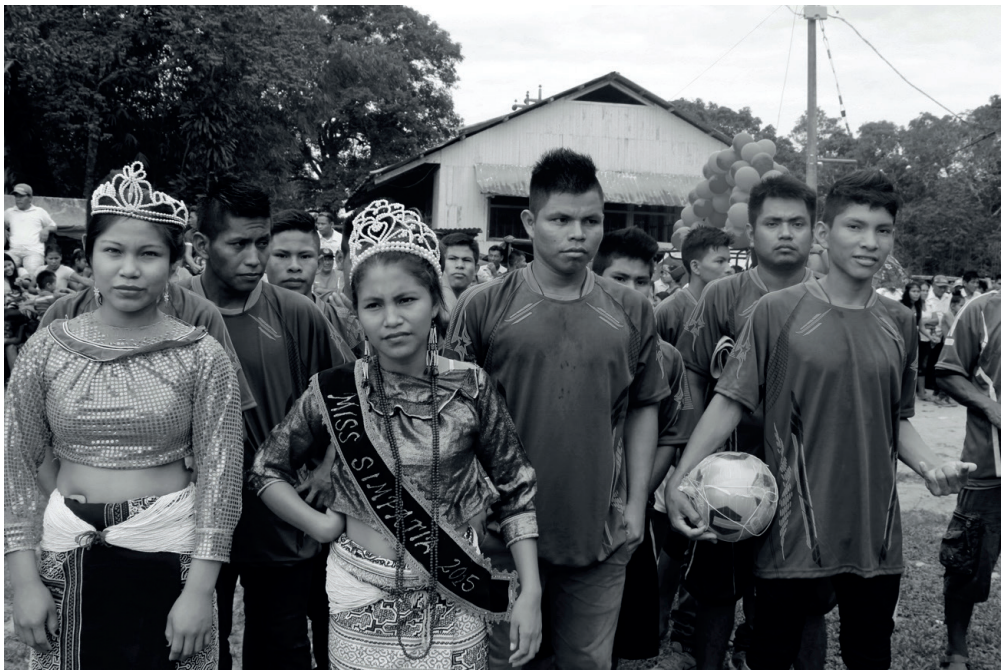

Foto 3. Desfile con reinas de belleza comunales (Foto de Sanny Ancón, 2016).

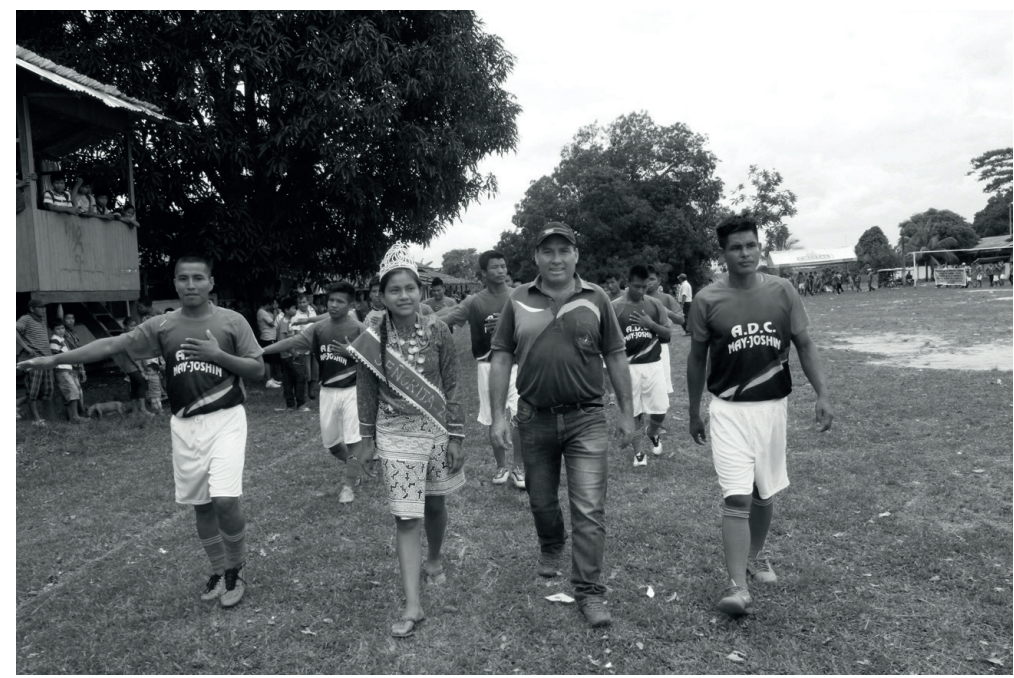

Foto 4. Desfile de inauguracion (Foto de Sanny Ancón, 2014).

Es importante señalar que el campeonato de futbol femenino ocurre primero (ver fotos 5 y 6), y una vez que este termina, viene el campeonato masculino, en el que los equipos van eliminándose hasta llegar a la final. Según el reglamento del campeonato, los partidos de mujeres duran 50 minutos, con dos tiempos de 25 minutos cada uno, y en caso de empate, se resuelve con penales. El partido final del campeonato femenino dura 60 minutos, con dos tiempos de 30 minutos cada uno, y en caso de empate, se juegan dos tiempos adicionales de 10 minutos cada uno; y 
si se mantiene el empate, se va al tiro con penales. En el caso masculino, los partidos regulares duran 80 minutos cada uno, con dos tiempos de 40 minutos, y si hay un empate, se resuelve con tipos de penal. La única excepción es el partido final, en el que se juegan 90 minutos, con dos tiempos de 45, y si hay empate, hay dos tiempos adicionales de 15 minutos adicionales, y si se mantiene el empate, se va a penales.

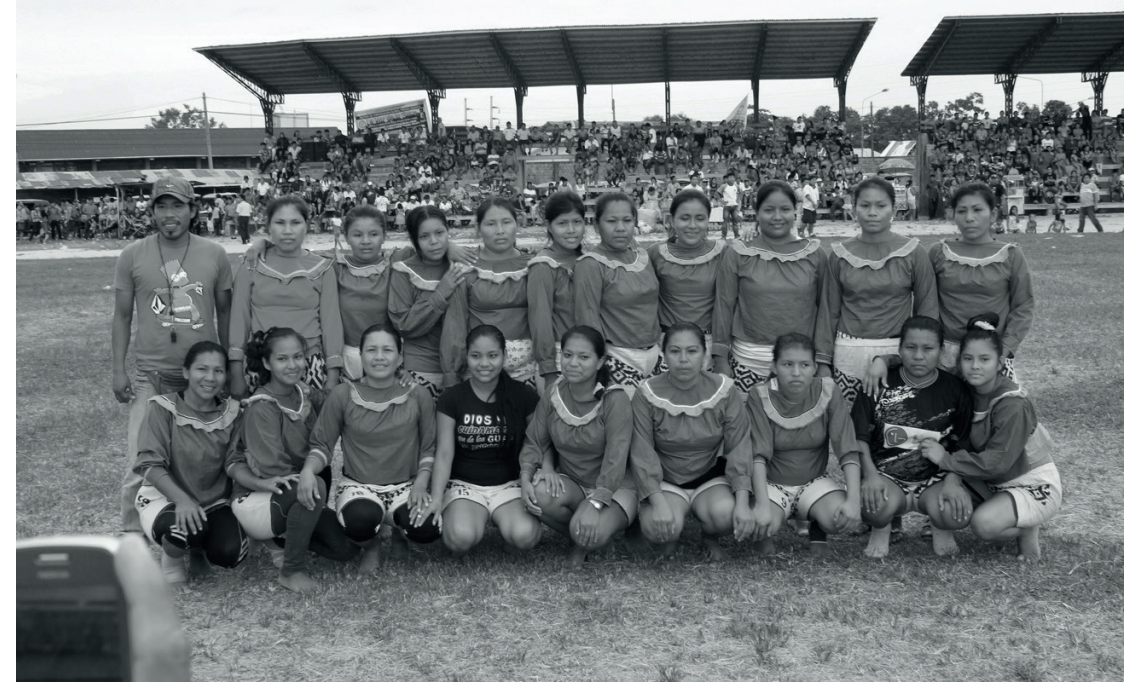

Foto 5. Equipo femenino (Foto de Sanny Ancón, 2014).

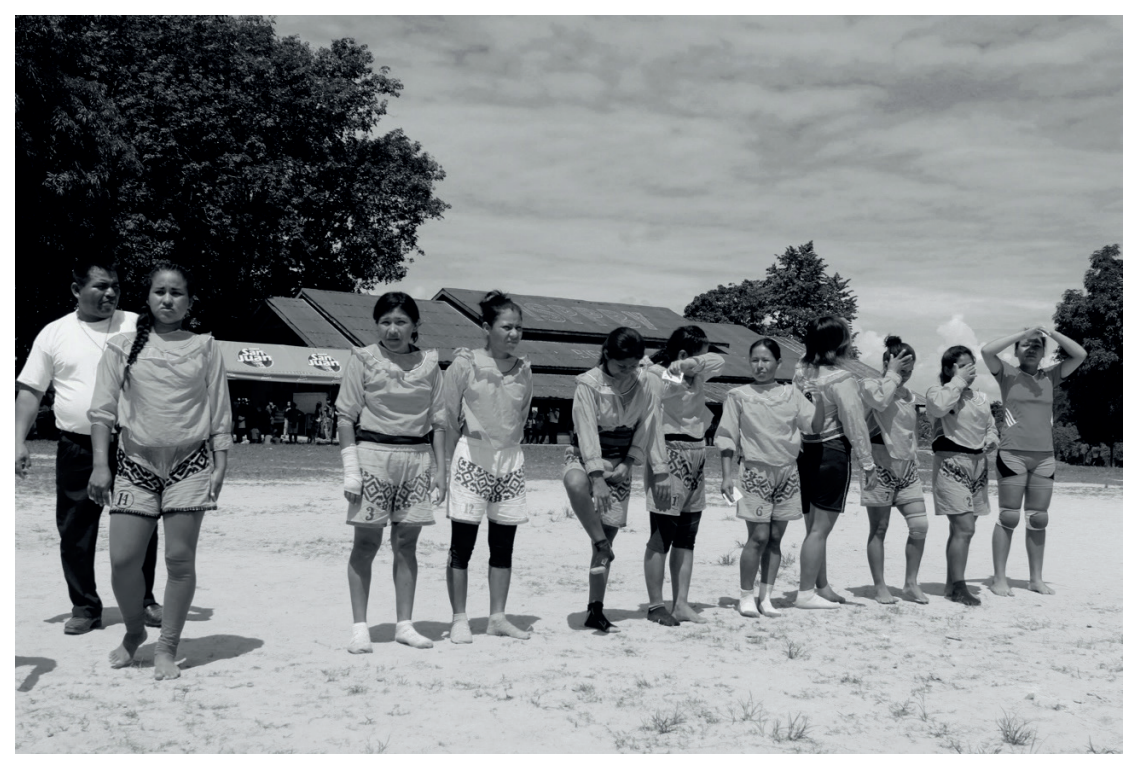

Foto 6. Equipo femenino antes de final (Foto de Sanny Ancón, 2016). 
Todos los partidos son narrados en castellano y shipibo por un equipo de locutores que se ubican en una caseta especialmente diseñada para ello (ver foto 7). Algunos de los locutores, además, se han ido especializando en esta tarea. Entre estos, se encuentran Dante Sánchez Martínez, Wikleff Yui López, Luis Márquez Pinedo e Ítalo García Murayari, con quienes trabajó la lingüista Maricielo Guillén (2018) en su tesis sobre locución deportiva en idioma shipibo; pero también hay otros locutores, como Ronald Suárez Maynas, el actual presidente del Consejo Shipibo Konibo Xetebo (COSHIKOX) y quien jugó un rol clave para la participación del equipo peruano en la «Copa América Indígena» o «Copa Americana de Pueblos Indígenas» que se celebró en Chile en el año 2015. En el caso del partido final, este también se transmite por radio en directo a través de FMStereo100 y también por streaming a través de internet.

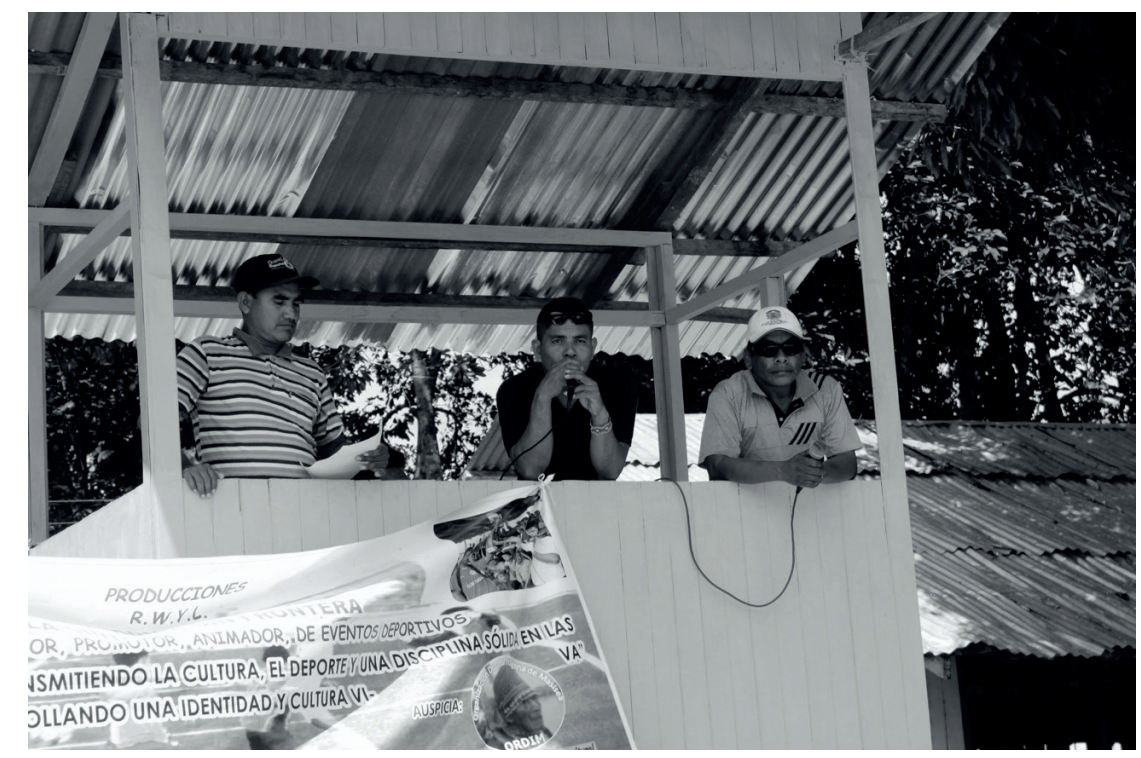

Foto 7. Cabina de locutores (Foto de Sanny Ancón, 2016)

Una vez que se juega el partido final, se procede a la clausura en la que se dan los premios a los equipos ganadores (ver foto 8). El equipo campeón, además de la copa, recibe un premio en efectivo, al igual que el equipo subcampeón, mientras que el tercer y cuarto lugar solo llevan medallas y diplomas. Finalmente, luego de la clausura y la entrega de premios, se tiene la fiesta final con música, comida y bebida. 


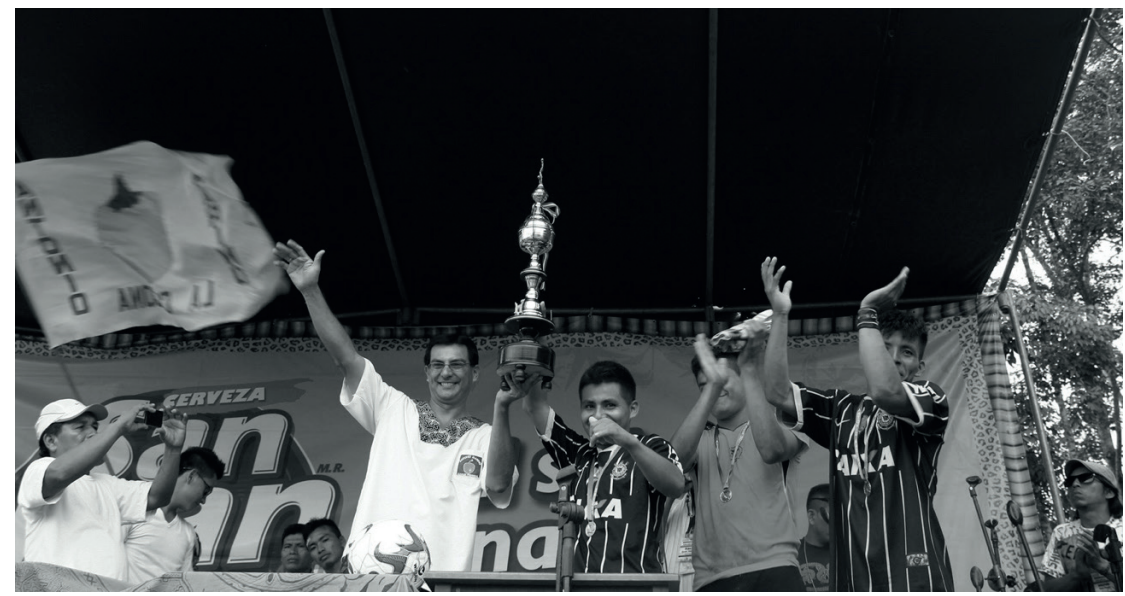

Foto 8. Premiación de ganadores (Foto de Sanny Ancón, 2014)]

Este momento final es muy importante, ya que desde un inicio el Mundialito ha convocado a mucha gente. La mayoría de personas shipibas coincide en que se trata, probablemente, del evento social más importante para este pueblo, convocando a familias de todas las comunidades de la región. Asimismo, muchas familias que viven en distintas ciudades del país aprovechan esta ocasión también para viajar a Pucallpa, y los barrios y asociaciones organizan también sus equipos para participar en el campeonato. En el año 2009, por ejemplo, el equipo de la comunidad urbana de Cantagallo de Lima llegó incluso a recibir apoyo del Instituto Peruano del Deporte para poder viajar hasta Pucallpa, y terminaron, además, como los campeones de ese año.

En un principio, el Mundialito era organizado por el propio ISPPBY y, por lo tanto, contaba con el apoyo institucional y logístico de esta institución. Posteriormente, conforme el campeonato fue creciendo, fue necesario crear una instancia más formal para ello. Así, en el año 2012 se creó la Asociación Indígena de Fútbol (AIF) para hacerse cargo del evento. Esta organización fue reemplazada en el año 2015 por la Asociación de Fútbol Indígena de la Amazonía y Afines (conocida como ASFIA o también AFIAA). Esta asociación, sin embargo, recibió muchas críticas, e incluso corrieron rumores de que los organizadores se habían llevado parte del dinero destinado al evento; por ello, desde el año 2016, la organización responsable del Mundialito es la Asociación de Fútbol Indígena del Perú (AFUIP), liderada en los últimos años por el profesor Abel Escobar.

A partir de 2016, las tensiones entre los dirigentes de la ASFIA, entre los que destaca Jonás Franco Anihuari, y los de la AFUIP, fueron creciendo. El resultado final fue la organización de dos campeonatos paralelos: el llamado «Mundialito 
tradicional» quedó a cargo de la AFUIP, mientras que Franco y la ASFIA comenzaron a organizar, a partir de 2019, la «Copa Amazónica Indígena». Esta última logró recibir el apoyo del gobierno regional de Ucayali y contar, gracias a este aporte, con un importante premio monetario. Sin embargo, este tiene menos acogida que el campeonato tradicional, y lo que terminó ocurriendo fue que los equipos que van quedando eliminados en este último pasan a inscribirse en el organizado por la ASFIA. Es importante señalar, además, que a diferencia del campeonato tradicional, que sigue teniendo como principal objetivo ser un evento festivo para compartir entre las comunidades shipibas, el de la ASFIA explícitamente busca promover un equipo shipibo para que juegue de manera sostenida en la Copa Perú. Sin embargo, antes de la existencia de la ASFIA ya han venido participando en este torneo nacional varios equipos shipibos, como el de la comunidad nativa Ceylán, que juega en la liga distrital de Masisea; el de la comunidad de San Francisco, que juega en la liga distrital de Manantay, y el club May Jushin, que juega en la liga distrital de Yarinacocha.

A lo largo del tiempo, además, el Mundialito se ha ido transformando y modernizando, buscando entrenadores, contratando a jóvenes shipibos que sobresalen por sus habilidades, y tratando de ser más profesionales. Algunos jóvenes que están estudiando en la ciudad de Pucallpa o de Lima, si son buenos jugadores, son contratados por las comunidades para reforzar sus equipos. Sin embargo, todos los jugadores o jugadoras deben ser indígenas. En este sentido, la pertenencia étnica es fundamental, ya que los equipos participantes tienen que representar a una comunidad nativa o a un club deportivo indígena. Según el reglamento del Mundialito: «Todos los jugadores deben pertenecer a un pueblo indígena con dominio de su lengua y la identidad con su pueblo o comunidad. En caso excepcional, si el jugador/a es descendiente de padre y madre indígena, debe tener dominio de la lengua originaria preferentemente de uno de los padres» (citado por Guillén 2018, pp. 29-30).

A lo largo del tiempo los shipibos también han ido creando clubes y asociaciones deportivas de carácter más permanente que buscan profesionalizar más este deporte. Entre estas, se encuentran la Asociación Deportiva Jepe Ian, la Asociación Deportiva Cultural Soi Niwe, la Asociación Deportiva Atlético Nacional Muy Jushin Club o la Asociación Deportiva Intercultural de la Selva Peruana (ADISFP). El más antiguo de estos clubes es el Jepe Ian, vinculado también a la Asociación Cultural del mismo nombre, fundada en 1991 por un grupo de profesores shipibos, entre los cuales se encontraba el profesor Evaristo López Maynas. Posteriormente, el equipo y la asociación cultural se separaron formalmente, y en la última década el club deportivo se ha ido formalizando más, contando entre sus directivos a Ronald Suárez Maynas, Edilberta Amasifuen Picota y Jean Esthefard Amasifuen Amasifuen, entre otros. El segundo en antigüedad es May Jushin, fundado por el profesor 
Abel Escobar a inicios del presente siglo. La creación de clubes deportivos indígenas con aspiraciones profesionales no solo ocurre entre los shipibos. Otros equipos indígenas que también se están profesionalizando son el Club Gavião Kyikatêjê de Bom Jesus de Tocantins, fundado en el año 2009, que es el primer equipo indígena en Brasil, o el Club Atlético Nauta (Acudecana), equipo kukama basado en la ciudad loretana de Nauta fundado en 2014.

El proceso de profesionalización del Mundialito incluye la adopción de las reglas de la FIFA, y de otros aspectos más formales, como el hecho de que los jugadores y equipos técnicos tienen que estar debidamente registrados y acreditados por los organizadores, y en los últimos años también se les exige el uso de carnés de identificación. Asimismo, los jugadores deben estar adecuadamente vestidos, siendo los organizadores más exigentes con los equipos masculinos que con los femeninos. Por otra parte, el estilo de juego también ha ido cambiando. Hoy en día entrenan de manera más profesional y bajo la dirección de profesores de educación física o de entrenadores capacitados. También se juega de una manera menos brusca. Y existe un interés explícito entre los jugadores por ser contratados por equipos profesionales, interés compartido por los organizadores y por los clubes y asociaciones deportivas. De hecho, ya desde hace algunos años, diversos equipos profesionales de la región envían observadores al Mundialito para reclutar a los mejores jugadores. A lo largo del tiempo, varios jugadores shipibos han formado parte de equipos como el Sport Loreto o el Deportivo Bancos; sin embargo, siempre han tenido problemas para desarrollar carreras profesionales, principalmente por problemas de talla o de técnica deportiva. De ahí también el interés de los clubes shipibos por mejorar el rendimiento físico y adquirir mejores técnicas de juego.

\section{El Mundialito como nuevo ani Xeati}

A pesar de que la práctica del fútbol se ha extendido por todo el mundo, y de que cada vez se organizan más campeonatos de fútbol indígena como el peladão indígena de Manaos, el campeonato de equipos indígenas de Tocantins, el «Intertribol» (campeonato estadual indígena de São Paulo, el campeonato indígena de Roraima, o el campeonato de fútbol indígena de la Amazonía organizado por la Organización ce los Pueblos Indígenas de Colombia (OPIAC), el éxito del «Mundialito shipibo» solo puede entenderse a cabalidad como continuación de las propias tradiciones shipibas. En este sentido, no puede entenderse como un acto mimético de una sociedad subalterna para apropiarse de la cultura hegemónica o resistir a ella. Una manera más exacta de entender el Mundialito partiría, por lo contrario, de verlo como la recreación de una fiesta importante de la propia tradición shipiba como es el ani xeati que dejó de celebrarse hace ya varias décadas, aunque en los últimos 
años ha habido distintos intentos de volver a celebrarlo, eliminando algunos de sus aspectos más cuestionables.

El ani xeati tradicional era una gran fiesta compuesta por una serie de rituales, siendo quizás el más conocido — y ciertamente el más polémico- el de la ablación o circuncisión del clítoris (xebijana tsekati) de las jóvenes púberes, de ahí que muchas veces se ha querido interpretar como un rito de pubertad femenino. Sin embargo, estudios más recientes corroboran que la xebijana tsekati no se celebraba únicamente en el contexto del ani xeati y que, además, el resto de rituales eran tan o más importantes que este último, y por ello tiene más sentido hablar de un ritual múltiple o de un mega ritual que expresaría y celebraría la identidad étnica shipiba. De hecho, el término ani xeati significa literalmente "la gran fiesta».

Los preparativos para esta gran fiesta comenzaban varios meses antes, con los viajes de comitivas especiales para invitar a la gente y con los preparativos logísticos para poder garantizar el alojamiento, el alimento y la bebida que iban a ser necesarios durante los varios días que esta iba a durar. La fiesta propiamente dicha empezaba con la bienvenida de los visitantes, y desde este momento inicial comenzaban la música y los cantos — de diverso tipo ${ }^{5}$ — que acompañaban a los distintos rituales que forman parte de la gran fiesta. Al inicio, también las personas que participaban de la fiesta se pintaban el rostro y distintas partes del cuerpo con diseños kené y otros, aspecto que a veces también se mantiene en el Mundialito (ver foto 9).

Luego de la bienvenida, se celebraban las peleas y enfrentamientos rituales de hombres contra hombres y de mujeres contra mujeres. En el caso de los varones, el enfrentamiento podía darse de manera verbal, desafiando a los que se iban incorporando a la fiesta, pero también a través de peleas con macanas (winon rishkiti). A veces a esta parte de la gran fiesta se le denominaba koshimeananai o "fuerceo» (forcejeo) ya que se medía la fuerza y las relaciones entre los distintos varones y mujeres. Posteriormente se realizaba también el enfrentamiento ritual entre varones que se caracterizaba por los cortes hechos en las nucas de los contrincantes con las navajas llamadas wexati. Este ritual en particular exigía el derramamiento de sangre, que así marcaba la reconciliación entre los dos varones enfrentados ${ }^{6}$.

\footnotetext{
5 Ruiz Urpeque (2016, p. 81) menciona los siguientes tipos de cantos que podían estar presentes en algún momento durante la fiesta: mashá, bewa, shiro bewa, nawarin, ai iká, ishori, y yoina peoti.

6 Algunos autores (Morin y Saladin D’Anglure, 2007 o Ruiz Urpeque, 2016) enfatizan este derramamiento de sangre masculino como un aspecto fundamental de la fiesta y como contraparte del ritual de pubertad femenino, donde la cliterodoctomía ocupaba un lugar central.
} 


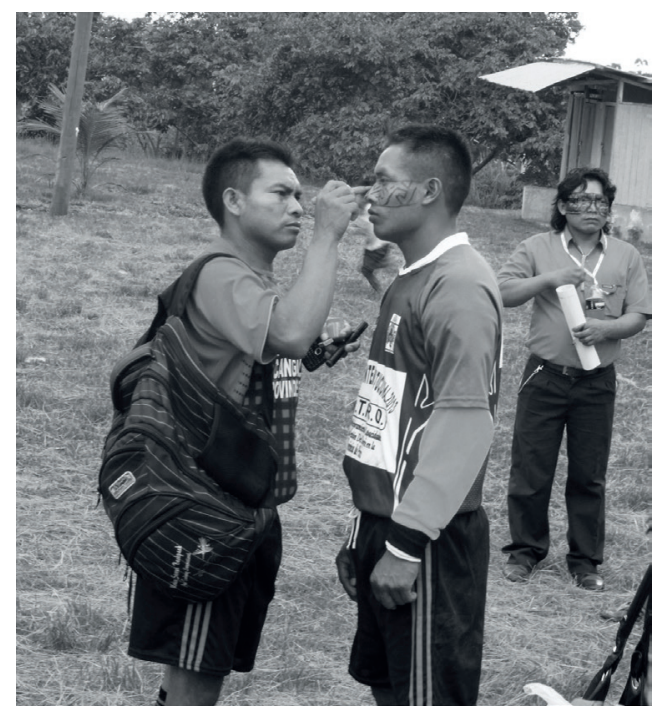

Foto 9. Pintándose el rostro con diseños tradicionales (Foto de Sanny Ancón, 2014).

En general, una de las principales razones, aunque no la única, de estos enfrentamientos - tanto entre hombres como entre mujeres - era resolver las tensiones que habían surgido a partir de episodios de adulterio o de celos. De manera similar, las mujeres se enfrentaban también, pero jalando, y eventualmente arrancando, mechones del cabello de su contrincante en lo que ahora se denomina como bachinananai o chobeo. Es importante señalar, como insiste Ruiz Urpeque (2016, pp. 54-55), que no se deben confundir ni la pelea entre varones (con macanas o wexati) ni la pelea entre mujeres (chobeo) con los forcejeos previos en los que se probaba la fuerza física y la fortaleza moral de los otros varones o mujeres.

Posteriormente, se celebraban los ritos de paso femeninos, en los que se simbolizaba la conversión de las niñas púberes en «verdaderas mujeres» (jakon ainbo) y, por lo tanto, en verdaderas personas shipibo (jakon jonibo). Este paso se hacía en dos momentos: el del bésteti o corte de cerquillo, y el del xebijana tsekati o cliterodoctomía, en el cual la joven o las jóvenes — en caso que hubiera más de una- eran embriagadas y operadas por mujeres especializadas en esta tarea. Esta parte del ritual tenía lugar de manera privada.

Otra parte importante de la fiesta consistía en el flechado ritual de animales silvestres que habían sido criados por la familia anfitriona. En este caso, todos los adultos varones tiraban flechas por turnos a los animales que estaban atados a unas estacas. Sin embargo, el objetivo de este ritual no era acertar y matar a la bestia — tarea que le correspondía propiamente al dueño de la casa—, sino la de acercarse lo más posible al animal sin darle, hasta que finalmente, luego de que todos habían 
tirado, el dueño de la casa lo hacía. Inmediatamente, las mujeres de la casa corrían a recoger al animal para asegurar su muerte, y luego se lo llevaban para luego ser cocinado e incluido como parte de la comida festiva.

Finalmente se concluía con la gran fiesta propiamente dicha, marcada por la abundancia de música, danzas, comida y masato (bebida de yuca fermentada). Finalmente, se tiene que subrayar que no todas las celebraciones del ani xeati incluían todos estas partes o rituales específicos, aunque sí podían estar presentes, y, además, no siempre se celebraban en el orden aquí descrito.

En los últimos años, como se ha mencionado, se han organizado en distintas ocasiones fiestas que explícitamente son llamadas ani xeati, aunque estas no corresponden exactamente a la versión tradicional debido a que se han cambiado o eliminado algunas partes o rituales, como el xebijana tsekati o las peleas con wexati, pero manteniendo otros, como el bestati, el flechado ritual de animales, y por supuesto, la fiesta propiamente dicha donde se come, se bebe y se baila. Uno de estos ani xeati fue organizado por la comunidad de San Francisco en el año 2014. Según los organizadores de este evento, el «ani xeati está dirigido principalmente para el pueblo shipibo-konibo para demostrar cómo organizaban nuestros ancestros, nuestros abuelos, nuestras abuelas, porque ani xeati era un encuentro familiar, un encuentro del pueblo, del medio del alto Ucayali, cuando alguien organizaba ani xeati, todo el mundo venía a participar de ese evento» (Rojas 2015, p. 11). Otro de los más importantes fue el organizado por COSHICOX en la comunidad de Caco Macaya, en el distrito de Iparía, en el año 2017.

El Mundialito recoge algunos aspectos claves del ani xeati, principalmente el espacio de convivencia y de encuentro festivo. Como se ha señalado antes, muchas familias shipibas viajan desde sus comunidades o desde distintas partes del país para estar presentes en Pucallpa durante las fechas del campeonato y poder reencontrarse con sus parientes (ver fotos 10 y 11).

Asimismo, las pruebas de fuerza y las peleas rituales, tanto de hombres como de mujeres, son reemplazadas ahora por los partidos de los campeonatos masculino y femenino en el que los y las jóvenes shipibos demuestran su fuerza corporal, así como otras habilidades y destrezas. En este sentido resulta importante recordar que para el pueblo shipibo una persona valiosa es una buena persona o jakon joni. Para ello, uno debe ser una persona que piensa correctamente y que tiene buen juicio, y por ello debe ser shinanya joni o shinan jakon joni. Pero también debe ser una persona fuerte, que tiene fortaleza, tanto física como moral, para enfrentar la vida y sus desafíos; es decir, ser koshi joni.

Algunos shipibos comparan también el ritual del flechado con los tiros de penal. Al igual que en el ritual tradicional, uno a uno, los distintos jugadores tienen la posibilidad de mostrar su destreza, ya no frente al animal que va a ser flechado, sino frente al arco del equipo rival. 


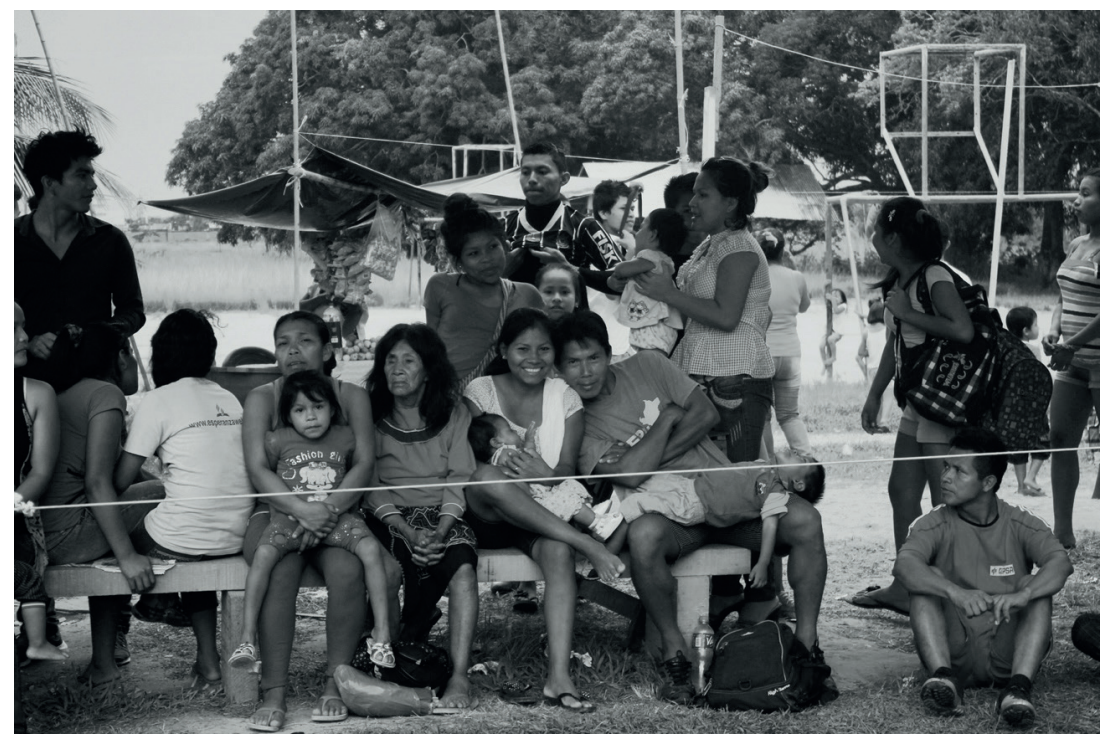

Foto 10. Familia indígena reunida durante Mundialito (Foto de Sanny Ancón, 2014).

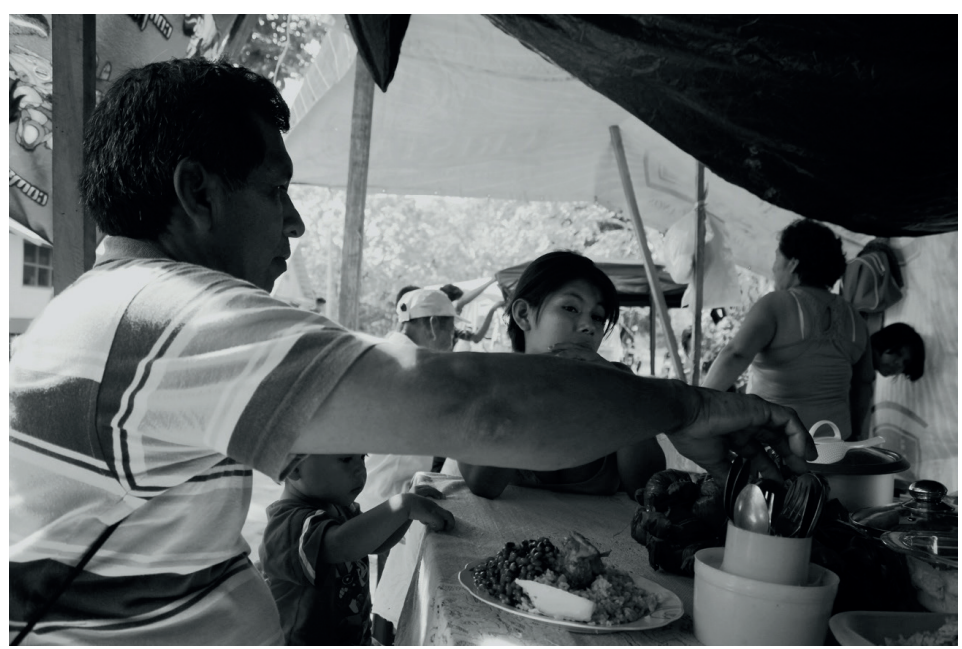

Foto 11. Compartiendo la comida (Foto de Sanny Ancón, 2014).

Finalmente, el Mundialito, al igual que el antiguo ani xeati, permite que los y las jóvenes shipibas puedan conocerse y establecer nuevas parejas (ver foto 12). No solo los jóvenes deportistas — hombres o mujeres — que demuestran sus habilidades y destrezas en el campo, sino también el resto de jóvenes que participan en el evento: las reinas de belleza, los locutores o los y las jóvenes que están en el público encuentran una oportunidad para conocer a potenciales parejas. 


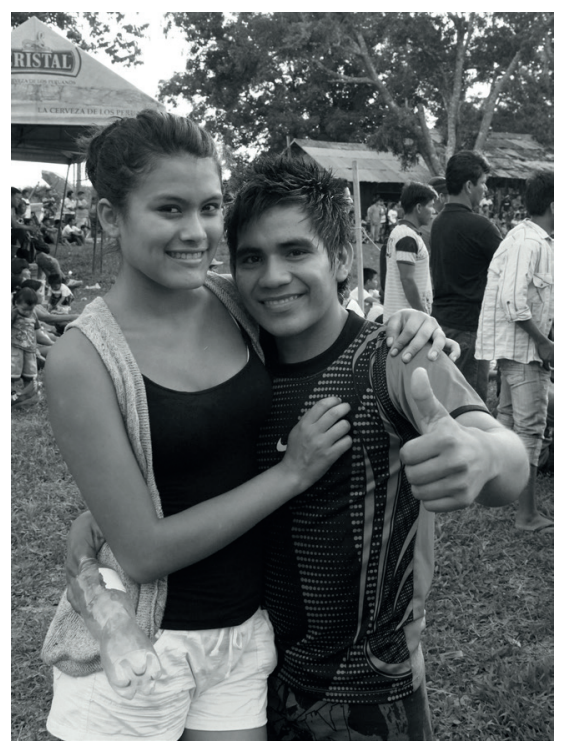

Foto 12. Pareja de jóvenes formada durante Mundialito (Foto de Sanny Ancón, 2014).

\section{Conclusiones}

A lo largo de los años, diversas personas, tanto shipibas como estudiosos de distinto origen, han expresado que el Mundialito es el nuevo ani xeati. Como señalaba el reconocido profesor Elí Sánchez en una de las inauguraciones del campeonato: "Hemos perdido el ani xeati y durante mucho tiempo no tuvimos otra manera de reencontrarnos. Ésta es la forma de hoy, como hacen otros pueblos en todo el mundo. Es tan importante que en estos días se suspenden las actividades productivas en las comunidades» (citado por Suárez 2011, p. 17).

La identificación entre el Mundialito y el ani xeati es muy común entre la gente shipiba, y prácticamente se ha convertido en una frase común hacer esta comparación, sobre todo entre los maestros bilingües, la gente mayor, o líderes y dirigentes. Así, por ejemplo, lo afirman por igual, el profesor Laurencio Ramírez (comunicación personal, 2016) o el presidente del Consejo Shipibo Konibo Xetebo (COSHIKOX), Ronald Suárez (comunicación personal, 2018), entre muchas otras personas.

El Mundialito ha sabido mantenerse, además, no solo como un espacio de encuentro y convivencia, sino también de reafirmación identitaria. De ahí que, aunque en el nombre oficial del campeonato se busque la inclusión de otros pueblos indígenas, sigue siendo fundamentalmente un evento shipibo: donde las familias y comunidades shipibas se reencuentran y donde se habla en shipibo, incluso por parte de los narradores de los partidos. 
Como se ha visto a lo largo del texto, el Mundialito también se ha ido transformando a lo largo del tiempo, buscando modernizarse y profesionalizarse. En este sentido, también cumple un rol político importante en la medida en que les permite expresar a los shipibos su identidad étnica bajo formas modernas y contemporáneas. Erikson (2013) se preguntaba si la forma de jugar el fútbol por los indígenas es una forma de «indigenizar la modernidad» o si, por el contrario, se trata de «occidentalizar a los indígenas». Al respecto, resulta más sugerente plantear el problema desde otra perspectiva, señalando de manera similar a la de Halbmayer (2018), que los pueblos indígenas son esencialmente modernos, no solo en la medida en que se han constituido como tales a partir del contacto colonial —ocurrido en la llamada Edad Moderna-, sino sobre todo por seguir configurándose en relación al Estado y al resto de la sociedad nacional y mundial.

Sin embargo, a pesar de ser un evento que se transforma constantemente, sigue siendo un espacio auténticamente shipibo. De ahí que el éxito del Mundialito tiene sentido si es que se le considera como una reelaboración del antiguo ani xeati, la gran fiesta del pueblo shipibo. En este sentido, antes que un acto mimético de resistencia o de apropiación de un elemento foráneo proveniente de la cultura hegemónica, corresponde más a la revitalización, en un contexto histórico nuevo, de valores y prácticas culturales propias de la sociedad shipiba. Por ello, no solo constituye un espacio donde se juega y se ve jugar fútbol, sino también es un momento privilegiado para que los parientes se reencuentren, los y las jóvenes se conozcan y formen nuevas parejas, y por supuesto, donde se come y se bebe juntos, se baila y se celebra la vida.

Se trata, pues, en última instancia, de un espacio ritual en el que la vida cotidiana entra en paréntesis para reafirmar el valor de la vida en común: la reconciliación de las diferencias, el establecimiento de alianzas y de relaciones de amistad, la continuidad generacional en la que se retoman las tradiciones del pasado y se incorpora a los y las jóvenes como parte del mismo colectivo, y, sobre todo, es el espacio en donde se actualiza y renueva el sentido de ser parte de un mismo pueblo.

\section{REFERENCIAS}

Acuña, Á. (2010). Estructura y función del fútbol entre los yanomami del Alto Orinoco. Revista Española de Antropología Americana, 40(1979), 111-138.

Almeida, A. J. M., Almeida, D. M. F. y Grando, B. S. (2010). As práticas corporais e a educação do corpo indígena: a contribuiçáo do esporte nos jogos dos povos indígenas. Revista Brasileira de Ciências do Esporte, 32(2-4), 59-74.

Andrade do Nascimento, J. R. y Faustino, R. C. (2009). Jogos indígenas: o futebol como esporte tradicional kaingang, Pensar a prática, 12(3), 1-12. 
Banet-Weiser, S. (1999). The Most Beautiful Girl in the World: Beauty Pageants and National Identity. Berkeley: University of California Press.

Berloffa Rodrigues, L. (2013). Notas introdutórias sobre o futebol enquanto elemento de sociabilidade indígena. Anais do VII Encontro Regional Sul de História Oral. Disponible en https://www.sul2013.historiaoral.org.br/resources/ anais/5/1377308712_ARQUIVO_artigoeventohistoriaoral.pdf

Berloffa Rodrigues, L. (2014). A prática do futebol entre indígenas de Dourados (MS). Tesis de Maestría en Historia. Dourados: Universidade Federal de Grande Dourados (UFGD).

Brito Vianna, F. (2001a). A bola, os «brancos» e as toras: futebol para indios xavantes. Tesis de Maestría en Antropología Social. São Paulo: Universidad de São Paulo.

Brito Vianna, F. (2001b). Olimpíadas indígenas exercitam o diálogo Intercultural. En C. A. Ricardo (ed.), Povos indigenas no Brasil-1996/2000 (pp. 218-222). São Paulo: Instituto Socioambiental.

Brito Vianna, F. (2008). Boleiros do cerrado: indios xavantes e o futebol. São Paulo: Annablume. Chiquetto, R. (2014). Entre índios e boleiros no Peladão Indígena. Ponto Urbe [online], 14. Consultado el 19 de abril 2019. Recuperado de http://journals.openedition.org/ pontourbe/1618. https://doi.org/10.4000/pontourbe.1618

Conklin, B. (1996). Reflections on Amazonian anthropologies of the body. Medical Anthropology Quarterly, 10, 373-5.

Dziubinska, M. (2013). Upiti kwaiti: Un idéal du football kakataibo (Amazonie péruvienne). Journal de la société des américanistes, 99(1), 183-194.

Eichberg, H. (1997). Body Cultures: Essays on Sport, Space, and Identity. Londres y Nueva York: Routledge.

Erikson, P. (2013). Une affaire qui roule? De l'introduction du football en Amazonie indigène. Journal de la société des américanistes, 99(1), 167-172.

Espinosa, O. (2017). Los pueblos shipibo-konibo, isconahua y kakataibo. Colección: Nuestros Pueblos Indígenas, n. ${ }^{\circ}$ 3. Lima: Ministerio de Cultura.

Fermino, A. L. (2012). O jogo de futebol e o jogo das relaçóes entre os Laklänó / Xokleng [tesis de Maestría em Educación]. Florianópolis: Universidad Federal de Santa Catarina.

Giulianotti, R. (1999). Football: A Sociology of the Global Game. Cambridge: Polity.

Grando, B. S. (2015). Jogo entre "parentes», os processos de educação do corpo, esporte e lazer indígena no Brasil: reflexóes a partir dos jogos dos povos indígenas. Revista Pedagógica, 17(34), 36-58.

Guillén, M. (2018). Hacia una tipología de las cláusulas en shipibo-konibo: evidencia desde la narración futbolística tesis de licenciatura en Lingüística]. Lima: Pontificia Universidad Católica del Perú.

Halbmayer, E. (ed.) (2018). Indigenous Modernities in South America. Canon Pyon: Sean Kingston Publishing.

Massa, G. L. (2009). El ritual del fútbol como medio de construcción de identidades masculinas en jóvenes de una comunidad nativa machiguenga [tesis de licenciatura en Antropología]. Lima: Pontificia Universidad Católica del Perú. 
McCallum, C. (2001). Gender and sociality in Amazonia: how real people are made. Oxford: Berg.

Morin, F. y Saladin D’Anglure, B. (2007). Excision feminine/incisión masculine ou la construction sociale de la personne chez les Shipibo-Conibo d'Amazonie Peruvienne. En N.-C. Mathieu (ed.), Une maison sans fille est une maison morte: la personne et le genre en sociétés matrilinéaires etlou uxorilocales (pp. 183-212). París: Editions de la Maison des Sciences de L'Homme.

Nascimento, R. D. (2015). O futebol Munduruku: um jogo estratégico nas relaçóes interétnicas e interculturais em Juara-MT [tesis de maestría en Educación]. Cuiabá: Universidad Federal de Mato Grosso.

Pires Rosse, E. (2013). Du foot en terres amérindiennes. Notes sur les cas a'uwẽ et tikmũ'ũn du Brésil. Journal de la société des américanistes, 99(1), 173-182.

Rojas, K. (2015). Noma Soi: Reinas de belleza en la cultura shipibo conibo: el caso de Cantagallo (Lima) y San Francisco (Pucallpa). Informe de investigación inédito.

Rolando, G. (2010). Dime cómo vienes y te diré quién eres: notas en torno al Estado en la Amazonía Indígena. Anthropia, 8, 48-54.

Ruiz Urpeque, E. (2016). Xebijana tsekati: contexto y significado de la clitoridectomía entre los Shipibo-Konibo del Ucayali [tesis de maestría en Estudios Amazónicos]. Lima: Universidad Nacional Mayor de San Marcos.

Schweitzer de Palacios, D. (2008). Shamanes, bellezas y Copa Mundial: de la selva al campo de fútbol. Anthropos, 103(1), 127-138.

Seeger, A., Da Matta, R., y Viveiros de Castro, E. (1979). A construção da pessoa nas sociedades indígenas brasileiras. Boletim do Museu Nacional, 32, 2-19.

Suárez Álvarez, C. (2011). La Edad del Desarrollo: señoritas y muchachos en la selva que se acaba [tesis de Maestría en Estudios Amazónicos]. Leticia: Universidad Nacional de Colombia.

Turner, T. (1995). Social body and embodied subject: bodiliness, subjectivity, and sociality among the Kayapó. Cultural Anthropology, 10, 143-70.

Vieira de Souza, M. (2014). Campeonato de Futebol "Peladão Indígena»: Um olhar sociocultural [tesis de maestría en Sociedad y Cultura en la Amazonía]. Manaos: Universidad Federal de Amazonas-UFAM.

Vilaça, A. (2005). Chronically unstable bodies: reflections on Amazonian corporalities. Journal of the Royal Anthropological Institute, 11(3), 445-464.

Vinha, M. (2004). Corpo-Sujeito Kadiwéu: jogo e esporte [tesis de Doctorado en Educación Física]. Campinas: Universidade Estadual de Campinas.

Walker, H. (2013). State of play: The political ontology of sport in Amazonian Peru. American Etnologist, 40(2), 382-398. 